concentrations of nifuratel obtained in the urine of treated subjects, so that the significance of our MIC findings for clinical practice is difficult to assess. The MICs of nifuratel of the three strains that were successfully eradicated from our patients were: $10 \mathrm{mg} / 1$ (C albicans), $25 \mathrm{mg} / 1$ ( $C$ tropicalis), and $50 \mathrm{mg} / 1$ ( $C$ stellatoidea). Most of the strains tested by us have had MICs in this range.

It seems reasonable to consider nifuratel as a possible choice for treating candidal urinary tract infection when the infection is thought to merit chemotherapy.

We are grateful to our colleagues, Mr D R Davies, Mr E Kirwan, and Professor A N Exton-Smith, for permission to treat patients under their care. Dr E Letley of Calmic Ltd and Dr A Salter of the Wellcome Foundation kindly supplied nifuratel tablets and powder. We are grateful for the meticulous care in specimen collection and follow-up shown by the nurses tending these patients.

\section{References}

1 Speller, D C E, fournal of Antimicrobial Chemotherapy, 1975, 1, 253.

${ }^{2}$ Nicholls, M W N, fournal of Medical Microbiology, 1970, 3, 529.

3 Speller, D C E, and Davies, M G, Fournal of Medical Microbiology, 1973, 6, 315.

4 Schönebeck, J, et al, Chemotherapy, 1973, 18, 321.

5 Grüneberg, R N, in Diagnosis and Chemotherapy of Urogenital Infections, Ed F Gasparri, p 109. Florence, Edizione Mediche P Periti, 1972.

6 McGeachie, J, Robinson, G, and Black, D, fournal of Clinical Pathology, $1972,25,447$.

7 Tynan, A P, Macis, F R, and Ward-McQuaid, J N, British fournal of Urology, 1969, 41, 271.

${ }^{8}$ Gower, P E, Clinical Trials fournal, 1970, 7, 389.

9 Brumfitt, W, in Diagnosis and Chemotherapy of Urogenital Infections, ed F Gasparri, p 411. Florence, Edizione Mediche P Periti, 1972.

\title{
Preoperative prediction of postoperative deep vein thrombosis
}

\author{
J K CLAYTON, J A ANDERSON, G P MCNICOL
}

British Medical fournal, 1976, 2, 910-912

\section{Summary}

A range of clinical data was obtained from 124 patients about to undergo operation and several coagulation tests were performed. No patient received prophylaxis for deep vein thrombosis, and isotopic scanning after operation showed that 20 patients had developed thrombosis. A simple prognostic index for predicting which patients would develop postoperative deep vein thrombosis was constructed using the clinical and coagulation data obtained before operation. The five variables with the best predictive power-euglobulin lysis time, age, presence of varicose veins, fibrin related antigen, and percentage overweight-produced an equation that identified $95 \%$ of those who developed deep vein thrombosis and misallocated only $28 \%$ of those who did not develop thrombosis. In view of the complications that low-dose heparin and dextran can cause, giving prophylaxis to under a third of the patients who will not develop deep vein thrombosis is clearly better than giving it to all.

\section{Introduction}

Recently impressive evidence has accumulated that the incidence of postoperative deep vein thrombosis can be reduced by prophylactic measures, particularly low-dose heparin and perioperative dextran 70.12 Both low-dose heparin and dextran may, however, significantly increase bleeding problems at the

Departments of Obstetrics and Gynaecology, Community Medicine and General Practice, and Medicine, University of Leeds, Leeds LS1 3EX

J K CLAYTON, MRCOG, senior registrar in obstetrics and gynaecology

$\mathrm{J}$ A ANDERSON, MA, DPHIL, (now reader in statistics, University of Newcastle upon Tyne)

G P MCNICOL, MD, PHD, professor of medicine time of surgery, ${ }^{34}$ and our object was to try to identify before operation those patients particularly at risk of deep vein thrombosis in the hope that in future it would be unnecessary to give prophylactic treatment to patients in a low-risk group.

\section{Patients and methods}

One hundred and twenty-four patients undergoing major gynaecological surgery, both abdominal and vaginal, were investigated. The following clinical data were obtained: age; weight; height; length of stay in hospital before operation; preoperative haemoglobin level; smoking habits; presence of varicose veins on clinical examination; history of venous thromboembolic disease; the nature of the operation; and whether surgery was for benign or malignant disease.

Laboratory methods-Platelets were measured on a Coulter thrombocounter. Platelet factor III release was measured by the method of Spaet and Cintron, ${ }^{5}$ fibrinogen by the method of Ratnoff and Menzie, ${ }^{6}$ antithrombin III by the method of Howie et al, ${ }^{7}$ factor VIII by a onestage method of Breckenridge and Ratnoff, ${ }^{8}$ euglobulin lysis time by the method of Nilsson and Olow, ${ }^{9}$ serum fibrin related antigen (FR antigen) by the method of Merskey et $a l,{ }^{10}$ plasminogen by the method of Alkjaersig et al, ${ }^{11}$ antiplasmin by the method of Howie et $a l,,^{12}$ activated partial thromboplastin time by the method of Langdell et al, ${ }^{13}$ and thrombin time by the method of Eichelberger. ${ }^{14}$ Platelet aggregation was measured to adenosine diphosphate (ADP) $1 \mu \mathrm{mol} / \mathrm{l}, 4 \mu \mathrm{mol} / \mathrm{l}$, and $10 \mu \mathrm{mol} / 1$; to collagen; and to adrenaline by the aggregometer method of Born and Cross. ${ }^{15}$

Diagnosis of deep vein thrombosis-All patients underwent preoperative isotropic scanning of the legs using the ${ }^{125}$ I-fibrinogen technique with the criteria of Kakkar. ${ }^{16}$ Routine postoperative scanning was performed on the first, third, and sixth days unless a significantly high count was obtained when daily scanning was started. None of the patients had received specific prophylaxis for deep vein thrombosis.

\section{Results and comment}

No patients had evidence of deep vein thrombosis before surgery. After operation 20 of the 124 patients developed isotopic evidence of deep vein thrombosis, which was confirmed by ascending venography in all of the 18 in whom this procedure was performed.

Clinical and coagulation data on the 104 patients without deep vein thrombosis after operation and the 20 with deep vein thrombosis are shown in the table. 


\section{STATISTICAL ANALYSIS}

The object of the statistical analysis was to produce a simple prognostic index that could be applied preoperatively to indicate those patients with a high chance of developing deep vein thrombosis. The 10 variables available preoperatively with the best chance of being useful in distinguishing between the positive and negative groups were (see table): (1) fibrinogen; (2) factor VIII ; (3) euglobulin lysis time; (4) FR antigen; (5) age; (6) length of preoperative stay; (7) percentage overweight for height $;^{17}(8)$ presence of varicose veins; (9) cigarette smoking habits, recorded as "Yes" or "No" for the month before surgery; and (10) benign or malignant disease, as confirmed by postoperative histology. Variables 8 and 9 were scored 0 for absence and 1 for presence. Variable 10 was scored 0 for benign and 1 for malignant. Subsequently, $x_{1}$ represents the value taken by variable $1, x_{.2}$ the value taken by variable 2 , and so on.

Significant preoperative clinical and coagulation data

\begin{tabular}{|c|c|c|c|}
\hline \multirow{2}{*}{\multicolumn{2}{|c|}{ Preoperative data }} & \multicolumn{2}{|c|}{ Postoperative leg scan } \\
\hline & & Positive $(n=20)$ & Negative $(n=104)$ \\
\hline \multicolumn{4}{|c|}{ Clinical data } \\
\hline Age (years) & . & $58=3$ & $44: 2$ \\
\hline "I) Overweight for height & . & $13: 3$ & $5: 1$ \\
\hline Preoperative stay (days) & & $4: 1$ & $2: 0.5$ \\
\hline Cigarette smoking (". of gro & up) $\quad \ldots$ & 25 & 48 \\
\hline Presence of varicose veins (", & of group) & 45 & 20 \\
\hline Malignant disease ("." of gro & up) $\quad \ldots$ & 20 & 1 \\
\hline \multicolumn{4}{|c|}{ Coagulation data } \\
\hline Fibrinogen $(\mathrm{g}$ l) $\quad \ldots$ & .. & $3 \cdot 33 \div 0 \cdot 13$ & $2.96 \div 0.09$ \\
\hline Factor VIII (" of normal) & $\therefore$ & $127: 7$ & $107: 3$ \\
\hline Euglobulin lysis time (min) & $\therefore$ & $412 \div 29$ & 270.14 \\
\hline Serum FR antigen (mg/) & . & $9: 1$ & $5 \div 1$ \\
\hline
\end{tabular}

Linear prognostic and diagnostic indices have been used successfully, ${ }^{1 \times 19}$ and we selected this approach for the present study. The method of logistic discrimination ${ }^{19}$ was used to estimate the coefficients of the prognostic index, I. All the above variables could have been fed into the system, but instead a more informative, stepwise approach was taken. The best single variable for prediction was found to be variable 3, euglobulin lysis time. Then the variable that added most to the prediction power of variable 3 was found to be variable 5 , age. Proceeding in this stepwise fashion, variables 8,4 , and 7 (varicose veins, FR antigen, and percentage overweight for height) were added sequentially, in that order. No other variable significantly increased the prognostic power so we decided to restrict the prognostic index to these five variables. In all cases the statistical criterion used was a log-likelihood ratio test. The prognostic index based on these five variables as estimated from the observations of the 124 patients in the study is given in the following equation:

$$
I=11 \cdot 3+0 \cdot 0090 x_{3}+0 \cdot 22 x_{4}+0.085 x_{5}+0 \cdot 043 x_{7}+2 \cdot 19 x_{8} .
$$

Large positive values of $\mathrm{I}$ are associated with a high risk of developing deep vein thrombosis and large negative values are associated with a low risk. Small values of I are equivocal.

This analysis does not imply that the variables not included in the equation are not prognostic for deep vein thrombosis. Rather, it means that after the information in the equation has been taken the remaining variables are redundant because they do not add anything further to the prognosis.

To find the value of the index, I, for a patient, the values of the five variables $(3,4,5,7$, and 8 ) were substituted into the equation. The values of I for the 124 patients in the study are presented in the figure. Clearly, patients with and without deep vein thrombosis were well separated. Nevertheless, there was inevitably a "grey" zone where

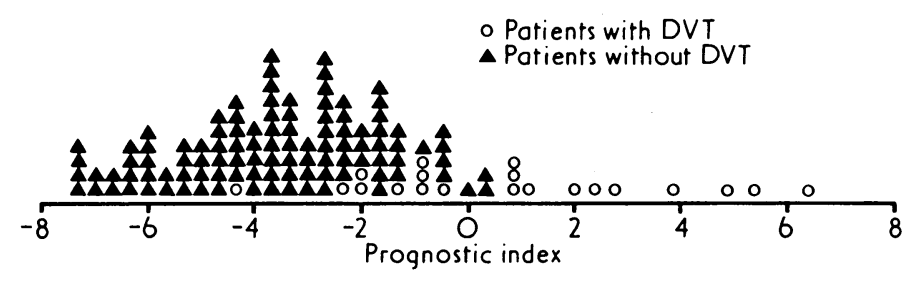

Prognostic index (I) for deep vein thrombosis (DVT). prediction was less certain. When the cut-off point was taken at $I=0,11$ of the patients who developed deep vein thrombosis were correctly predicted, while two patients without deep vein thrombosis were incorrectly allocated. When the cut-off point was taken at -2.5 all but one of the positive patients were correctly allocated but the number of patients without deep vein thrombosis who were incorrectly identified rose to 29 .

\section{Discussion}

Our results show that, by using three pieces of simple clinical information and the results of two laboratory tests, acceptable preoperative prediction of postoperative deep vein thrombosis is possible. Depending on the balance of importance attached to giving unnecessary prophylaxis to patients with a low risk of developing deep vein thrombosis and not treating patients with a high risk, a point on the scale can be selected to determine which patients should receive prophylactic treatment. At present the balance of benefit might suggest a cut-off point at $-2 \cdot 5$, which would identify $95^{\circ}{ }_{0}$ of patients who will develop deep vein thrombosis, at the expense of unnecessarily treating $28^{\circ}$ " of those who will not. This is clearly a vast improvement on the outcome of routine universal prophylaxis-that is, the "unnecessary" treatment of all patients who will not develop deep vein thrombosis.

The data suggest that the addition of the laboratory data improved the separation and justified the extra effort needed.

Before the isotopic technique for diagnosing deep vein thrombosis became available, Hume and Chan ${ }^{20}$ compared the results of several haematological tests performed postoperatively in 29 patients who had clinical evidence of postoperative deep vein thrombosis with those in a control group of preoperative patients. Using a stepwise discriminant analysis, they derived a discriminant function from platelet adhesiveness, packed cell volume, and partial thromboplastin time. Later Hume et al, ${ }^{21}$ with a computer programme analysing clinical data, Doppler flowmeter assessment, a small panel of laboratory test results, and venography, correctly diagnosed thrombosis in $88^{\circ}{ }_{0}$ of the patients studied only after operation.

These studies from Hume and his associates differ from ours in that our prediction and classification was based on preoperative data in patients confirmed not to have deep vein thrombosis before surgery. Laboratory test results in blood taken after thrombosis have occurred may be a consequence of thrombosis or of surgery rather than related to predisposition to deep vein thrombosis.

Our approach is inevitably somewhat biased towards favourable results - the index was derived from our own patients and then reapplied to them-and it is essential to carry out a prospective study to examine the performance of the method with the same equation when applied to a new population of patients. Nevertheless, this approach to the selection of high-risk patients has considerable promise.

We are grateful to Professor J S Scott for his interest in this work and for giving us access to patients under his care. Mr K R Peel also kindly permitted study of his patients. Financial support was received from the Board of Governors of the United Leeds Hospitals and the Special Trustees of the General Infirmary at Leeds. We are grateful to Miss V Binns for help with computing and to Mrs B Griffin and Mrs G Fieldhouse for laboratory work.

\section{References}

1 International Multicentre Trial, Lancet, 1975, 2, 45.

2 Bonnar, J, and Walsh, J, Lancet, 1972, 1, 614.

3 Gallus, A S, et al, New England fournal of Medicine, 1973, 288, 545.

${ }^{4}$ Multi-Unit Controlled Trial, Lancet, 1974, 2, 118.

5 Spaet, T H, and Cintron, J, British fournal of Haematology, 1965, 11, 269.

${ }^{6}$ Ratnoff, O D, and Menzie, C, Fournal of Laboratory and Clinical Medicine, $1951,37,316$.

${ }^{7}$ Howie, P W, Prentice, C R M, and McNicol, G P, British fournal of Haematology, 1973, 25, 101.

${ }^{8}$ Breckenridge, R T, and Ratnoff, O D, Blood, 1962, 20, 137. 
${ }^{9}$ Nilsson, I M, and Olow, B, Acta Chirurgica Scandinavica, 1962, 123, 247.

${ }^{10}$ Merskey, C, Kleiner, G J, and Johnson, A J, Blood, 1966, 28, 1.

11 Alkjaersig, N, Fletcher, A P, and Sherry, S, fournal of Clinical Investigation, 1959, 38, 1086.

12 Howie, P W, et al, Lancet, 1970, 2, 1329.

${ }^{13}$ Langdell, R D, et al, fournal of Laboratory and Clinical Medicine, 1953, 41, 637.

14 Eichelberger, J W, jun, Laboratory Methods of Blood Coagulation, p 63. New York, Harper and Row, 1965.
15 Born, G V R, and Cross, M J, fournal of Physiology, 1963, 168, 178.

${ }^{16}$ Kakkar, V V, Archives of Surgery, 1972, 104, 152.

17 Geigy, Scientific Tables, 7th edn, p 712. Macclesfield, Geigy Pharmaceuticals, 1970

18 Anderson, J A, Biometrika, 1972, 59, 19.

10 Anderson, J A, et al, Quarterly fournal of Medicine, 1972, 41, 175.

${ }^{20}$ Hume, M, and Chan, Y K, Fournal of the American Medical Association, $1967,200,747$.

${ }^{21}$ Hume, M, Glancy, J J, and Chan, Y K, Archives of Surgery, 1968, 97, 894.

\title{
Virus-like particles in paraspinal muscle in scoliosis
}

\author{
J N WEBB, W J GILLESPIE
}

British Medical fournal, 1976, 2, 912-913

\begin{abstract}
Summary
Biopsy material from the skeletal muscle (paraxials) of 21 patients with scoliosis was examined by light and electron microscopy. Virus-like particles, $17 \mathrm{~nm}$ in diameter with a crystalline structure, were identified in the skeletal muscle fibres of four patients. Associated changes in the sarcoplasm included swelling of mitochondria, presence of lipid droplets, and vesicular structures. Serological studies and culture for virus isolation gave negative results. An excess of lipid (predominantly in type 1 fibres) was noted in the skeletal muscle of several other cases. The significance of these findings is obscure, but the morphology of the paraxial muscles of patients with scoliosis and controls is currently being investigated in greater detail.
\end{abstract}

\section{Introduction}

Idiopathic scoliosis is a crippling and distressing disorder of childhood, most cases occurring in young girls. Pathological studies of the musculoskeletal system have not succeeded in elucidating the cause of the condition. ${ }^{1}$ Scoliosis secondary to neurological disease or myopathic processes is well recognised, ${ }^{2}$ and it is therefore tempting to speculate that an undisclosed disorder affecting muscles of the spine might be responsible for causing the condition. Nevertheless, clinical $^{3}$ and traditional histological methods have failed to show any important abnormalities. Hirano ${ }^{4}$ in an electronmicroscopical study has described degenerative changes in the back muscles of patients with idiopathic scoliosis. It was suggested on the basis of these findings that a myopathic process might be implicated in the aetiology of the condition.

\section{Patients and methods}

Through the kindness of colleagues at the Princess Margaret Rose Orthopaedic Hospital, Edinburgh, we have had the opportunity of

Department of Pathology, Western General Hospital, Edinburgh J N WEBB, MD, FRCPED, consultant pathologist

Department of Orthopaedic Surgery, Princess Margaret Rose Hospital, Edinburgh

W J GILLESPIE, MB, FRCSED, consultant orthopaedic surgeon examining biopsy specimens of skeletal muscle (taken from paraspinal muscles: rotators and multifidus) from 21 patients with scoliosis; 12 had idiopathic disease, seven had congenital scoliosis, and two had neurofibromatosis. At open exposure of the spine for intra-articular fusion, or at revision, blocks of muscle tissue were taken from each side from the deeper parts of the paraspinal muscles at the apex of the curve. Frozen sections were cut for histological and histochemical study, and $1-\mathrm{mm}^{3}$ pieces of tissue were fixed in $2.5 \%$ buffered glutaraldehyde for electron microscopy.

\section{Results}

No definite histological abnormality was observed in the muscle on light microscopy and no appreciable changes were seen in the type 1 and type 2 fibres, as shown by the dihydronicotinamide adenine dinucleotide diaphorase and myosin adenosine triphosphatase (at $\mathrm{pH}$ 9.4) methods. In some cases a slight excess of intracellular lipid was noted, mainly in type 1 fibres. This was confirmed on electron microscopy (fig 1).

A wholly unexpected finding in four patients (see table) was the presence of virus-like particles within the muscle fibres (fig 2). They consisted of uniform electron-dense particles aggregated into a

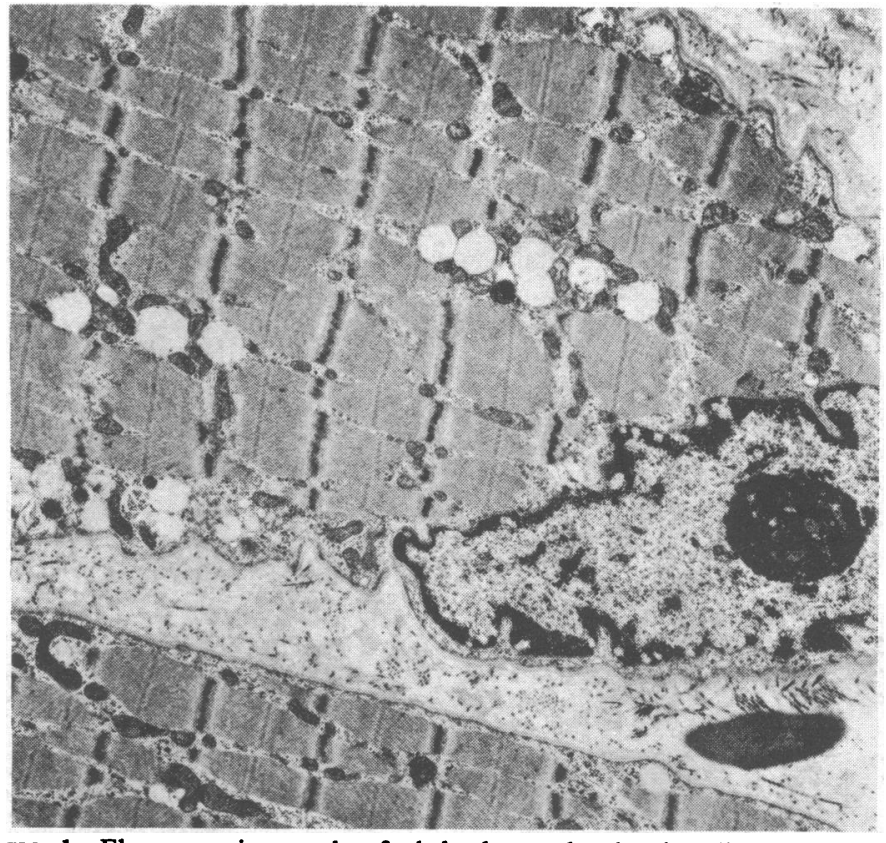

FIG 1-Electron micrograph of skeletal muscle showing lipid droplets between myofibrils and beneath sarcolemmal membrane. ( $x$ 5957.) 